\title{
Riesgos ambientales en Misiones: primera aproximación desde una perspectiva social del riesgo*
}

\author{
Environmental risks in Misiones: first approach from a social perspective of risk
}

\section{Sandra Pereira}

Magister de la Universidad de Buenos Aires (UBA). Instituto de Geografía "Romualdo Ardissone". Departamento de Geografía. Facultad de Filosofía y Letras. Universidad de Buenos Aires. Puán 480 (1406) Ciudad Autónoma de Buenos Aires, Buenos Aires, Argentina, sanper@filo.uba.ar

\section{Resumen}

El objetivo de este trabajo es realizar el estudio del principal riesgo ambiental de Misiones: las inundaciones, desde la perspectiva teórica de la construcción social del riesgo. En este marco teórico se acuerda con la premisa de que la suma de acciones, decisiones e inacciones que se toman cotidianamente en el territorio constituyen un proceso que conduce a la generación de un riesgo en tanto anticipación del desastre. Siendo también en este marco que se manifiestan las dificultades de la Gestión de Riesgo -GR-. Las inundaciones constituyen la principal causa de desastres en Argentina y Misiones es una de las provincias más afectada del país por los excesos hídricos. Debido a la escasa disponibilidad de publicaciones actualizadas sobre el tema, se utiliza información periodística obtenida on line, desde 2012 hasta fines de 2019, con el registro y análisis de la ocurrencia de inundaciones, sus causas y consecuencia, y el accionar de los agentes involucrados, para observar en forma crítica la coyuntura de la GR, que se evidencia con la asistencia en la emergencia.

Palabras clave: Gestión de Riesgo -GR-; Teoría social del riesgo; Ámbito rural; Misiones

\section{Abstract}

The objective of this work is to carry out the study of the main environmental risk of Misiones: the floods, from the theoretical perspective of the social construction of risk. In this theoretical framework, it is agreed with the premise that the sum of actions, decisions and inactions that are taken daily in the territory constitute a process that leads to the generation of a risk while anticipating the disaster. Being also in this framework that the difficulties of Risk Management -GR- are manifested. Floods are the main cause of disasters in Argentina and Misiones is one of the most affected provinces in the country due to water excesses. Due to the limited availability of updated publications on the subject, journalistic information obtained online has been used, since 2012 to the end of 2019, with the recording and analysis of the occurrence of floods, their causes and consequences, and the actions of the agents involved, to observe critical of the situation of the GR, which is evidenced by emergency assistance.

Key words: Risk Management -GR-; Social theory of risk; Rural area, Misiones

\footnotetext{
* El presente artículo es el inicio de una investigación mayor que se desarrollará en el marco del Proyecto FILO: CyT (FC19: 085) "Riesgos hidrometeorológicos, producción familiar y vulnerabilidad. Casos de estudio en la provincia de Misiones (Argentina)"
}

Cita sugerida: Pereira, S. (2020). Riesgos ambientales en Misiones: primera aproximación desde una perspectiva social del riesgo. Estudios Socioterritoriales. Revista de Geografía, (27), 051. DOI: //https://doi.org/10.37.838/unicen/est.27-051 


\section{INTRODUCCIÓN}

Las inundaciones representan la principal causa de desastres naturales en Argentina $y$ la región del Noreste -NEA- es la más afectada del país. Según información del documento "Análisis Ambiental de País"1, el 60\% de los desastres naturales en Argentina son producidos por las inundaciones y estas explican el 95\% de las pérdidas económicas y poblaciones afectadas. En 2016 se calcula que a nivel nacional se perdieron casi un millón y medio de dólares y más de setenta mil personas fueron afectadas por inundaciones. Además, en esta región y en casi el $80 \%$ de los casos, las lluvias causan el desborde de los principales ríos que forman la Cuenca del Plata (Paraná, Bermejo, Pilcomayo, Uruguay, Paraguay y sus afluentes).

La provincia de Misiones se encuentra en la segunda zona del país en la que se produce una mayor cantidad de precipitaciones -pp- (luego de los Andes Patagónicos), registrándose 1.800 $\mathrm{mm}$ por año, en temporadas normales, con importantes inundaciones estacionales (Mapa 1). Según datos del Servicio Meteorológico Nacional -SMN- en el período 1981-2010, las pp anuales en Misiones, alcanzaron los 1800 $\mathrm{mm}$, con unos 120 días de lluvia en el promedio, lo que la constituye en la provincia en la que se registró la mayor cantidad de lluvias a nivel nacional ( $\mathrm{SMN}, \mathrm{s} / \mathrm{f})$.

Ante esta situación, Misiones posee un plan de emergencia, adherido al Sistema Nacional, con la finalidad de prevenir desastres, basado en la alerta temprana y la protección civil, "para fortalecer la capacidad de respuesta". Sin embargo, institucionalmente se sostiene que los temporales superan las estimaciones, tienen un desarrollo mucho más rápido que el esperado, y las pp son mayores a las previstas (El Territorio, 14/10/2018).

Las actividades económicas desarrolladas en

1 Presentado en 2016 por el Banco Mundial y citado en el nuevo Plan Nacional para la Reducción del Riesgo de Desastres 2018-2023, dependiente del Sistema Nacional para la Gestión Integral del Riesgo (SINAGIR), en el marco del Ministerio de Seguridad y la Jefatura de Gabinete de Nación. el agro misionero son llevadas adelante por productores familiares, que constituyen una de las principales y más importante característica de esta provincia. Con pequeñas explotaciones, trabajadas casi exclusivamente con mano de obra familiar, con uno o más cultivos agroindustriales (yerba mate, té y tabaco) destinados a la venta en los secaderos locales y con huerta y granja para garantizar la reproducción familiar, Misiones es la 'provincia de los productores familiares'.

La Agricultura Familiar -AF- en Argentina es un sector representativo de la producción de alimentos y fibras textiles, destinadas tanto al mercado interno como externo, creando empleo en el espacio rural y ocupación del territorio. Obschatko en uno de sus últimos trabajos, calcula que la AF del país abarca unas 250 mil explotaciones agropecuarias -EAP's-, lo que es igual al 75\% del total de EAP's, que ocupan un escaso $15 \%$ (en promedio) de la superficie en producción, emplean casi el 60\% de la mano de obra directa y aportan poco más del $20 \%$ del valor de la producción (Obschatko, 2009). Tal vez la contribución económica puede parecer relativamente limitada, sin embargo esta varía según la actividad productiva considerada y la zona del país. Estos agricultores familiares representan la casi totalidad de los productores en cultivos como el tabaco, la yerba mate, el algodón, la caña de azúcar (cultivos agroindustriales); la gran mayoría de los productores hortícolas y frutícolas; y también más del $75 \%$ de los productores de cultivos extensivos (maíz y soja). En Misiones, en donde la presencia de productores familiares es prácticamente exclusiva, "estos generan el $51 \%$ del VBP superando al promedio nacional" (Craviotti, 2014, p. 7-8), y los hogares con necesidades básicas insatisfechas (NBI) alcanzan un porcentaje del 15,6\% (DINREP, 2014).

La magnitud de las frecuentes inundaciones provocadas tanto por pp abundantes como por crecidas de ríos y arroyos, y la situación de vulnerabilidad que puede reconocerse en la población afectada, tanto rural como urbana en Misiones, hace que el estudio de esta 
temática resulte de gran interés. Sobre todo en el marco de la teoría social del riesgo que profundiza la mirada sobre las cuatro dimensiones del riesgo, como la vulnerabilidad, la peligrosidad, la exposición y la incertidumbre; dimensiones que no pueden considerarse en forma separada si se quiere visualizar una so- lución integrada a este problema complejo. (Barrenechea et. al., 2000).

Es por todo lo anterior que el objetivo de este trabajo es estudiar la ocurrencia de las inundaciones en esta provincia desde la perspectiva teórica de la construcción social del riesgo, con el fin de hacer una contribución al tema.

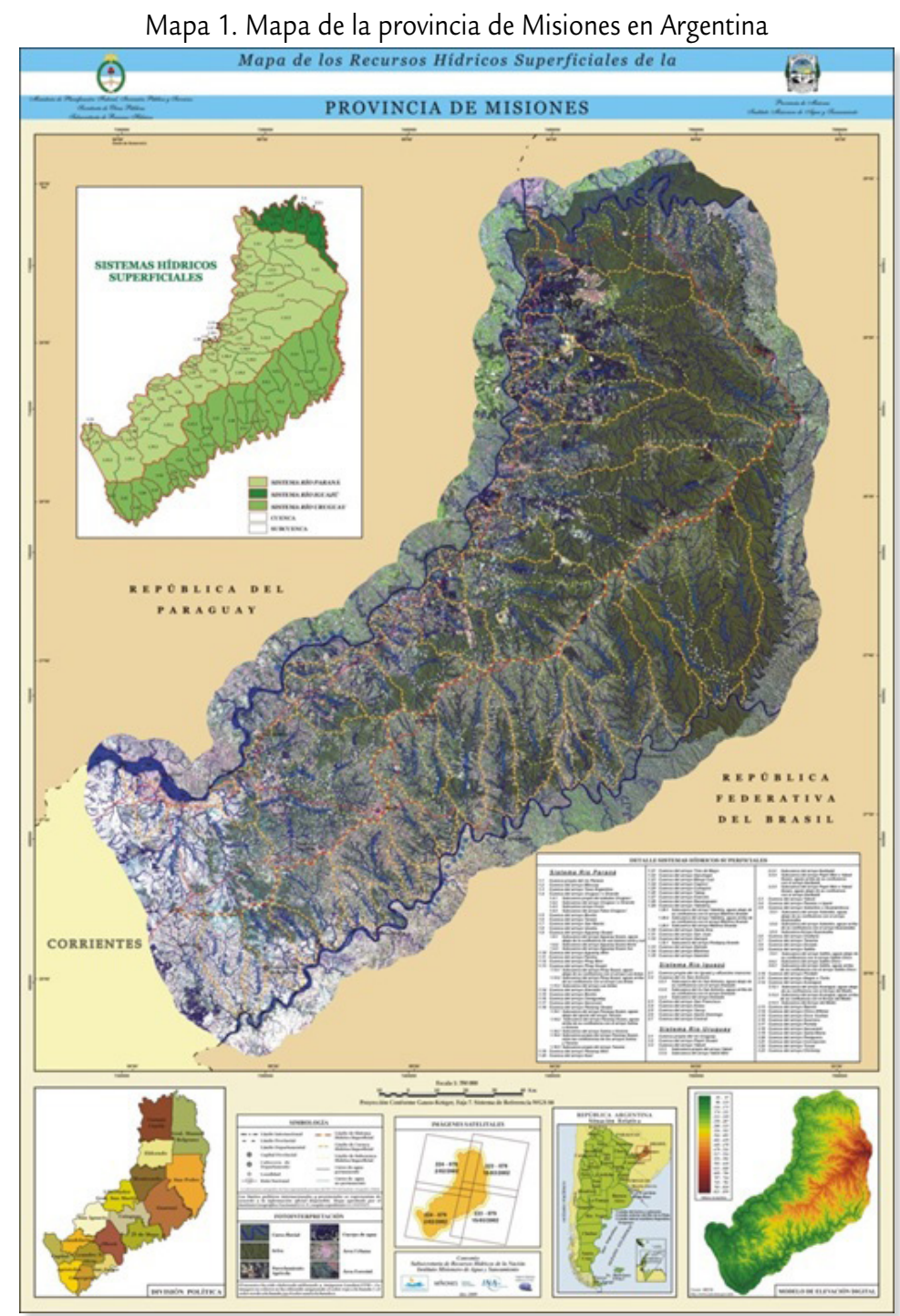

Fuente: https://www.argentina.gob.ar/sites/default/files/000_misiones_.jpg

\section{APROXIMACIÓN A LA TEORÍA SOCIAL DEL RIESGO}

En el marco de la Teoría social del riesgo se acuerda con la premisa de que la suma de acciones, inacciones y decisiones que se toman cotidianamente sobre el territorio constituyen un proceso que conduce a la generación de un riesgo en tanto anticipación del desastre (Beck, 2008), y por ello también se considera que se puede intervenir en cualquier momento para prevenir y mitigar los daños a futuro.

La catástrofe o el desastre tiene lugar en una sociedad determinada y en un momento histórico dado. Es así como las catástrofes solo 
pueden comprenderse como parte de un marco social más general: la sociedad moderna, una sociedad del riesgo (Beck, 2008). Una sociedad que 'construye' sus propios riesgos, los que aparecen activados ante la ocurrencia de eventos naturales y/o antrópicos que en solamente algunos casos son extremos. Una sociedad que tiene una vulnerabilidad definida por la situación social general anterior al evento y que parece ponerse a la vista ante la ocurrencia de aquel suceso que plantea una situación 'extraordinaria'. Una sociedad que en función de su propia vulnerabilidad dará distintos tipos de respuesta frente a la catástrofe o el desastre, anticipándose al mismo, actuando luego de su ocurrencia y/o previniendo a futuro, entre otras.

En este contexto la vulnerabilidad social es entendida como la situación económica y social en general, preexistente al evento catastrófico. Se define como "...la potencialidad que cada grupo social tiene de sufrir heridas o daños (...) y forma parte del riesgo en tanto que exista certeza por parte de los grupos sociales respecto a cuáles son los peligros que corren" (Natenzon, 2005, s/p).

Un componente central de la vulnerabilidad lo constituyen "los niveles de organización e institucionalización de los planes de mitigación (preparación, prevención y recuperación)”. Desde este punto de vista la vulnerabilidad está "directamente asociada al desarrollo", siendo caracterizada por los aspectos institucionales y culturales (Barrenechea, et. al., 2000, p. 3).

La idea de que el riesgo ${ }^{2}$ se despliega en la normalidad, implica la aceptación de una particular configuración de situaciones de vulnerabilidad social que predisponen a esa sociedad a experimentar los efectos negativos de una determinada peligrosidad. El riesgo es un “...término relativo y específico que implica siempre una cierta vulnerabilidad ante una amenaza específica" (Natenzon, 2005, s/p). Por lo tanto, vul-

2 Siguiendo a Barrenechea et. al. (2000) las cuatro dimensiones del riesgo son: la vulnerabilidad, la peligrosidad, la exposición y la incertidumbre. Las autoras sostienen que "considerar en manera aislada cualquiera de las cuatro dimensiones que constituyen el riesgo, con prescindencia de las otras, cierra el camino a la solución integrada de un problema complejo" (p. 2). nerabilidad social y peligrosidad son dos de los conceptos más ampliamente aceptados para analizar los riesgos de desastre. Es fundamental incorporar los aspectos o causas de fondo (Blaikie et. al, 1996) de la construcción de las situaciones de vulnerabilidad que definen el desastre ya que en la actualidad, a nivel internacional, se reconoce que el incremento del grado de exposición de las personas y los bienes, es más rápido que la disminución de la vulnerabilidad; lo que genera nuevos riesgos y un aumento constante de las pérdidas tanto económicas como sociales $y$ ambientales a nivel local ${ }^{3}$.

Otra de las dimensiones del riesgo es la incertidumbre que se relaciona con las

...limitaciones en el estado del conocimiento (in-
certidumbre técnica) y las indeterminaciones en
cuanto a competencias institucionales y aspec-
tos normativos (incertidumbre social). Al mismo
tiempo, estas limitaciones y la complejidad del
fenómeno en cuestión impiden el manejo de la
totalidad de las variables involucradas, impreg-
nando de incertidumbre los procesos de toma de
decisiones. (Barrenechea et. al. 2000, p. 2)

Tanto Natenzon (2005) como Barrenechea et. al. (2000) sostienen que las cuatro dimensiones del riesgo son: la vulnerabilidad, la peligrosidad, la exposición y la incertidumbre; y que considerar de manera aislada a cualquiera de ellas, impide la solución integrada de un problema que evidentemente es complejo. En este caso de estudio: las inundaciones que afectan a la población de Misiones en la actualidad.

Las fuentes de información en este caso son los principales periódicos de la provincia y de la CABA -Ciudad Autónoma de Buenos Aires-. Se recopiló, analizó y comparó la información periodística publicada y se obtuvieron los datos sobre la ocurrencia de los eventos climáticos, sus magnitudes y afectación; así como también

3 En el contexto internacional, el Marco de Sendai para la Reducción del Riesgo de Desastres propuesto para 2015-2030 por la $\mathrm{ONU}$, se reconoce como el instrumento que indica una línea de acción para reducir la cantidad de damnificados y víctimas, y las pérdidas económicas en infraestructura y servicios básicos. En los términos de formulación de dicho marco también se reconoce que todos estos eventos naturales se han exacerbado con el Cambio Climático, considerándolo como uno de los factores que promueve el riesgo de desastres (UNISDR, 2015). 
los organismos y agentes involucrados, en tiempo real. La información periodística se obtuvo por internet, recurriendo a las publicaciones de El Territorio, Misiones On Line, Primera Edición, Misiones Cuatro, Noticias del 6, Minuto Uno, Visitemos Misiones, El Tribuno y Clarín, La Nación, Infobae e Infocampo (de la CABA). Todo esto permitió un acercamiento a la situación histórica y actual de los territorios en los que tienen lugar dichos eventos.

\section{Organismos y noRmativas VIGENTES EN GESTIÓN DE RIESGO - GR}

Antes de comenzar con el análisis de la información periodística que es la fuente fundamental de este artículo es necesario presentar cuáles son los organismos públicos que se dedican a la GR en Argentina, con el objeto de poder comprender cómo funciona la GR de desastres desde el punto de vista institucional.

\section{El Sistema Nacional para la Gestión Integral del RiesGo y la PROTECCIÓN CIVIL - SINAGIR-}

En el año 2016 se creó el SINAGIR con el objeto de

...integrar las acciones y articular el funcionamiento de los organismos del Gobierno nacional, los Gobiernos provinciales, de la Ciudad Autónoma de Buenos Aires y municipales, las organizaciones no gubernamentales y la sociedad civil, para fortalecer y optimizar las acciones destinadas a la reducción de riesgos, el manejo de la crisis y la recuperación. (Art. 1, Ley 26509/09).

EI SINAGIR contó desde su comienzo con un Consejo Nacional y un Consejo Federal para la Gestión Integral del Riesgo -GIR-y la Protección Civil, ambos constituidos por representantes de diferentes organismos públicos, con diversas funciones e injerencias.

Este Sistema se rige de acuerdo a los lineamientos estratégicos y metas que se establecen en el Plan Nacional para la reducción del riesgo y la Protección Civil -PLANGIR-y sus correspondientes Programas Operativos Anuales para la GIR y la Protección Civil -POAGIR-. Cuenta con financiamiento del Fondo Nacional -FONAE- $y$ recursos del Presupuesto General de la Nación ${ }^{4}$.

En este marco normativo se aclara que la GIR ...es un proceso continuo, multidimensional, interministerial y sistémico de formulación, adopción e implementación de políticas, estrategias, planificación, organización, dirección, ejecución y control, prácticas y acciones orientadas a reducir el riesgo de desastres y sus efectos (...) comprende acciones de mitigación, gestión de la emergencia y recuperación. (Ley 27287)

El proceso operativo de la GIR tiene los siguientes componentes: a) análisis de riesgo; b) mitigación; c) prevención; d) preparación; e) planificación e instrumentación de modelos locales de desarrollo sustentable; y f) gestión de la respuesta.

Sistema Nacional para la Prevención y MitigaCIÓN dE EMERGENCIAS Y DESASTRES AGROPECUARIOS -SNPYMED-

EI SNPYMED se constituye en la principal herramienta de GR para el agro con cobertura en todo el país, siendo totalmente independiente del SINAGIR ${ }^{5}$. Con su origen en la Ley de Emergencia Agropecuaria -LEA-, este Sistema se creó

...con el objetivo de prevenir y/o mitigar los daños causados por factores climáticos, meteorológicos, telúricos, biológicos o físicos, que afecten significativamente la producción y/o la capacidad de producción agropecuaria, poniendo en riesgo de continuidad a las explotaciones familiares o empresariales, afectando directa o indirectamente a las comunidades rurales. (Art. 1, Ley 26.509/2009)

Este Sistema tomó las bases de la LEA e incorporó en su letra la prevención y mitigación, integrando medidas correctivas y prospectivas

4 El Estado Nacional, a través del Ministerio de Seguridad reviste el carácter de Fiduciante con el destino exclusivo e irrevocable previsto en la Ley y de acuerdo con las condiciones que se establezcan en el contrato de fideicomiso. El Fiduciario es el BNA -Banco de la Nación Argentina- por lo cual se constituye en administrador de los bienes que se transfieren en fideicomiso. El Estado Nacional, las Provincias y la CABA en tanto hayan adherido al SINAGIR, se constituyen como Beneficiarios del FONAE. El Tesoro Nacional se constituye como Fideicomisario. El fideicomiso tendrá una duración de 20 años, renovables (Art.25 al 31, Ley 27287, Sistema Nacional para la Gestión Integral del Riesgo y la Protección Civil, 28 de septiembre de 2016). 5 Según consta en la Ley $N^{\circ} 27.287$. 
de GR; así como también especificando que asistirá a explotaciones familiares o empresariales. Es por esto que puede ser considerado como un 'nuevo' marco legal, que tiene como objetivo superar la respuesta ante la ocurrencia de una emergencia o desastre, con acciones previas que puedan mejorar la situación de los afectados (en este caso identificados y definidos como EAP's familiares o empresariales) y sus producciones.

EI SNPyMED aplica sus directrices a través de la Dirección Nacional de Emergencia y Desastres Agropecuarios -DNEyDA-, que funciona en el Ministerio de Agroindustria de la Nación. En esta Dirección se encuentran el Consejo Consultivo de Emergencia Agropecuaria (presidido por un representante del Ministerio de Agroindustria) y la Comisión Nacional de Emergencias y Desastres Agropecuarios -CNEyDA- que se convoca en los casos en que los efectos negativos del evento ocurrido son de gran magnitud y/o amplia afectación territorial, siendo la que propone al Poder Ejecutivo la declaración de emergencia/desastre agropecuario, delimitando la zona afectada y el período de tiempo definido para cada situación, en función de la declaración previa realizada por la provincia afectada. El accionar de esta Comisión es llevada adelante por medio de una Secretaría Técnica Ejecutiva constituida para tal fin, que también observa la evolución de las emergencias declaradas y el control del cumplimiento de las medidas que se adopten.

Para financiar los programas y acciones propuestos el SNPyMED dispone de los recursos del Fondo Nacional para la Mitigación de Emergencias y Desastres Agropecuarios -FONEDA-, que desde el 2009 cuenta con un total de $\$ 500$ millones por año, provenientes de Rentas Generales. El $20 \%$ de este Fondo se debe destinar por ley a obras de prevención y/o mitigación, que deben ser solicitadas formalmente por medio de proyectos elaborados por los damnificados con el apoyo de las autoridades locales.

Una vez declarada la emergencia o desastre agropecuario, el Ministerio de Agroindustria se encarga de gestionar y asignar los Fondos y el personal requeridos para asistir a los productores y a los organismos públicos nacionales, provinciales o municipales encargados de realizar las acciones de ayuda. En la etapa de recuperación, finalizada la emergencia/desastre, se contempla por ley que este Ministerio y/o los Estados provinciales, municipales o comunales, sigan sosteniendo las actividades productivas de la zona afectada.

El proceso que deben seguir los productores perjudicados por algún evento extremo se inicia con la entrega al gobierno local de una declaración jurada en la que especifican las características de su explotación, de su producción y la afectación de la misma. Si el evento es de una magnitud tal que abarca varios municipios el pedido se tramita desde los gobiernos locales a la provincia y si son varias las provincias afectadas se solicita la declaración de emergencia o desastre a nivel nacional ${ }^{6}$. Es fundamental aclarar que este Sistema promueve la organización de los afectados para la formulación de los proyectos, que posteriormente financiará, y que estarán a cargo de la DNEyDA, para llevar adelante aquellas acciones que permitan prevenir los daños que pueden ocasionar los eventos extremos futuros.

\section{MISIONES Y EL SINAGIR}

Siguiendo las normativas establecidas por el SINAGIR, Misiones es la tercera provincia que se incorpora al mismo, lo que permite la asistencia automática de la Nación en caso de ocurrencia de eventos extremos y la posibilidad de solicitar recursos para trabajar en prevención (Misiones Online, 19/6/2018).

...los funcionarios provinciales entregaron la copia del proyecto de Ley de adhesión al SINAGIR que será tratado en la apertura de sesiones de la

6 Por ejemplo, en el caso que una inundación cubra el 50\% o más de un campo la situación del mismo se considera "Emergencia" y si se encuentra por encima del $80 \%$ o más es "Catástrofe". En cualquiera de los dos casos los beneficios son: prórroga o exención de impuestos inmobiliarios, diferimiento de vencimientos de préstamos y el otorgamiento de créditos blandos a los productores que lo soliciten. 
Cámara a partir del 1 de mayo y que cuenta con amplio consenso para su aprobación. El nuevo sistema permite articular la colaboración de los recursos nacionales y provinciales en los lugares afectados por emergencias y desastres... (Ministerio de Seguridad, 13/3/2018)

En este contexto el 2 de octubre de 2008, se realizó una reunión en Mendoza, en la cual las autoridades nacionales del SINAGIR se reunieron con los directores de defensa civil de distintas provincias (de Neuquén, Catamarca, Chaco, Corrientes, Entre Ríos, La Pampa, Jujuy, Misiones, Mendoza, Salta, Santa Fe, Río Negro, Tierra del Fuego, Tucumán y Buenos Aires) para trabajar en prevenir y afrontar las emergencias y posibles desastres. De dicho encuentro participaron el subsecretario de Operaciones de Protección Civil, el secretario de Articulación Científico Tecnológica y las autoridades de los ministerios del Interior, Defensa, Servicio Meteorológico Nacional y de la Cámara Argentina de Mediana Empresa (CAME) $y$ entre las actividades realizadas procedieron a la presentación del Plan Nacional de Reducción de Riesgo de Desastres (2018-2023) ya mencionado7.

Por su parte, en la provincia de Misiones, en el 2016 se creó la Subsecretaría de Protección Civil, en el marco del Ministerio de Gobierno de la Provincia, desde donde se afirma que es "la institución encargada de resguardar a la población ante la posibilidad de un desastre mediante la Gestión Integral de Riesgos”. En este ámbito se creó también el Plan Provincial de Emergencias y Desastres Climáticos, integrado por organizaciones gubernamentales, militares y no gubernamentales, para definir, planificar y articular las acciones para prevenir, mitigar, dar respuesta, rehabilitar y contribuir a la reconstrucción funcional ${ }^{8}$.

7 Al mismo tiempo, en el marco del Plan Provincial de Emergencias y Eventos Climáticos, la Subsecretaría de Protección Civil del Ministerio de Gobierno, está realizando diversas actividades para incorporar los conceptos del Centro de Operaciones de Emergencias -COE- a la Protección Civil. Desde el SINAGIR y de la Secretaría de Protección Civil del Ministerio de Seguridad de la Nación se promueve el establecimiento de los lineamientos a aplicar en el que caso de situaciones reales de emergencias en Misiones.

8 http://www.proteccioncivil.misiones.gob.ar/index.php/institucional/
El gobierno de la provincia por medio de la misma Subsecretaria y el Instituto Provincial de Loterías y Casinos Sociedad del Estado, puso en marcha el Programa de Ayuda Climática -PACdirigido a las familias afectadas por eventos climáticos, que consiste en el otorgamiento de créditos para la compra de muebles y electrodomésticos por única vez, por montos de 35 mil y 70 mil pesos, previa certificación de la Subsecretaria de Protección Civil.

La página web de esta Subsecretaría de Protección Civil permite registrar las denuncias de la población y tramitar el pedido de asistencia; además de ofrecer asesoramiento con protocolos de acciones a seguir ante la ocurrencia de inundaciones, tormentas e incendios, entre otros.

Cabe agregar que en la ciudad de Posadas, y dependiente de su municipalidad, en 2010 se organizó la Oficina de Prevención de Riesgos ante Fenómenos Naturales -OPAD-, junto con el Sistema de Alerta Temprana -SAT-, con el objeto de registrar los fenómenos meteorológicos, el índice de sequedad ambiental y las crecientes del río Paraná y de los arroyos que surcan esta ciudad. En la OPAD se centralizan y coordinan los informes provenientes de instituciones oficiales y privadas, nacionales y extranjeras, siendo la única dependencia de la provincia que brinda el SAT y la prevención ante fenómenos naturales.

Desde la Comuna capitalina queremos orientar de esta manera a los vecinos a través de las informaciones que diariamente vamos emitiendo, a los fines que la gente se vaya familiarizando con este tipo de información y la use para su beneficio. (OPAD, 2010)

La OPAD ${ }^{9}$ además de publicar esta información

9 Por medio de la creación de la OPAD, Posadas integra el grupo de "Ciudades Resilientes" de las Naciones Unidas e integrantes de la Plataforma Nacional para la Reducción de los Desastres. La ONU también distinguió a la ciudad como Municipio del mes de mayo 2014 por las tareas que viene desarroIlando la OPAD. En setiembre de 2014 la ONU seleccionó a los Municipios de Posadas y Encarnación (Paraguay) para comenzar a trabajar de manera conjunta, en Prevención ante fenómenos naturales. También la OPAD recibió el reconocimiento de la Honorable Cámara de Representantes de la provincia de Misiones y del Honorable Consejo Deliberante de Posadas. Desde el 25 de febrero de 2016 el Ejecutivo Municipal elevo el rango de esta dependencia a Dirección General, optimizando 
diaria del clima de la provincia (temperaturas mínimas y máximas, probabilidades de ocurrencia de tormentas, chaparrones, granizo, etc.), formula la advertencia que grafica en colores según la situación y pronóstico realizado, para promover la comprensión precisa de la información.

Esta oficina es la encargada de responder en el momento en que ocurre un evento y la responsable de promover, planear y mantener la coordinación y el trabajo conjunto entre los involucrados, con el objetivo de responder a las situaciones de emergencias. De este modo coordina el empleo de los recursos humanos y materiales del Estado en las etapas de mitigación, contención y reconstrucción.

En el mismo marco, son los Intendentes municipales, dentro de su jurisdicción territorial, quienes tienen a su cargo la planificación, organización, promoción, coordinación, control y dirección de la Junta de Defensa Civil, y, eventualmente, puede dirigir las operaciones de emergencia (Gobierno de Misiones, 03/10/2018).

LA ACCIÓN EN LA GR EN EL AGRO MISIONERO: LA DECLARACIÓN DE EMERGENCIA AGROPECUARIA

En enero de 2012 Misiones fue incluida en el grupo de provincias que forman parte del SINAGIR. El gobernador de la provincia firmó un decreto que fue aprobado por la CNEyDA, con la información requerida (datos agrometeorológicos y económicos que requieren el relevamiento de campo) (Noticias del 6, 20/01/2012).

Ese mismo año desde la Nación se incluyó a Misiones en la declaración de emergencia agropecuaria, pero esta vez por sequía y se dispuso

sus funciones y compromiso social. Por el mismo decreto se renombro la OPAD como Oficina de Prevención de Riesgos ante Fenómenos Naturales. El 13 de octubre 2016 en el acto por el Día Internacional de la Reducción de Riesgos por Desastres, se habilito la aplicación de la OPAD para celulares. http://opad. posadas.gov.ar/quienes_somos.php que la provincia tuviera 30 días para recabar la información y acceder a los beneficios que otorgue la Nación. Los productores enviaron sus declaraciones juradas, detallando las pérdidas sufridas, que fueron posteriormente verificadas. Como resultado se declaró la emergencia, que rigió por 180 días y estuvo sujeta a la evaluación de la CNEyDA ${ }^{10}$.

Dos años después, en noviembre de 2014 el entonces Ministerio de Agricultura, Ganadería y Pesca de la Nación declaró la emergencia y/o desastre agropecuario en Misiones por el desborde de los ríos Uruguay e Iguazú y por exceso de precipitaciones, por un período de 30 días, incluyendo a las EAP's dedicadas a la producción ganadera bovina, porcina, aviar, piscícola, frutihortícola, de caña de azúcar, forestal y de yerba mate de los municipios de San Vicente (que a fines de octubre había sido afectado por intensas ráfagas de viento, granizo y lluvia que arrasaron los galpones y plantas de tabaco); Concepción de la Sierra y Santa María, Itacaruaré, San Javier, Mojón Grande, Florentino Ameghino, Aurora, Alba Posse, 25 de Mayo y El Soberbio. Además de algunos municipios de San Pedro, Oberá, Gral. Belgrano, Leandro N. Alem, Apóstoles, Gral. San Martín, San Ignacio, Cainguás y Candelaria; afectando a unas 7 mil ha en total. En esta oportunidad los bancos nacionales, oficiales y/o mixtos y la Administración Federal de Ingresos Públicos -AFIP- arbitraron los medios para que los productores agropecuarios comprendidos en esta declaración pudieran recibir los beneficios previstos en la Ley $N^{\circ} 26.509$ (El Territorio, 15/11/2014).

A esta actuación a nivel Nacional, le precedió el accionar provincial con la declaración de zona de Desastre y Emergencia Económica, con la evacuación de más de 6 mil afectados (Mapa 2).

10 En este caso no se encontraron instrumentos legales (boletines oficiales de la Nación o de Misiones) que pudieran confirmar esta declaración. 
Mapa 2. Declaración de Emergencia Agropecuaria en Misiones en 2014 (25 jun - 24 dic)

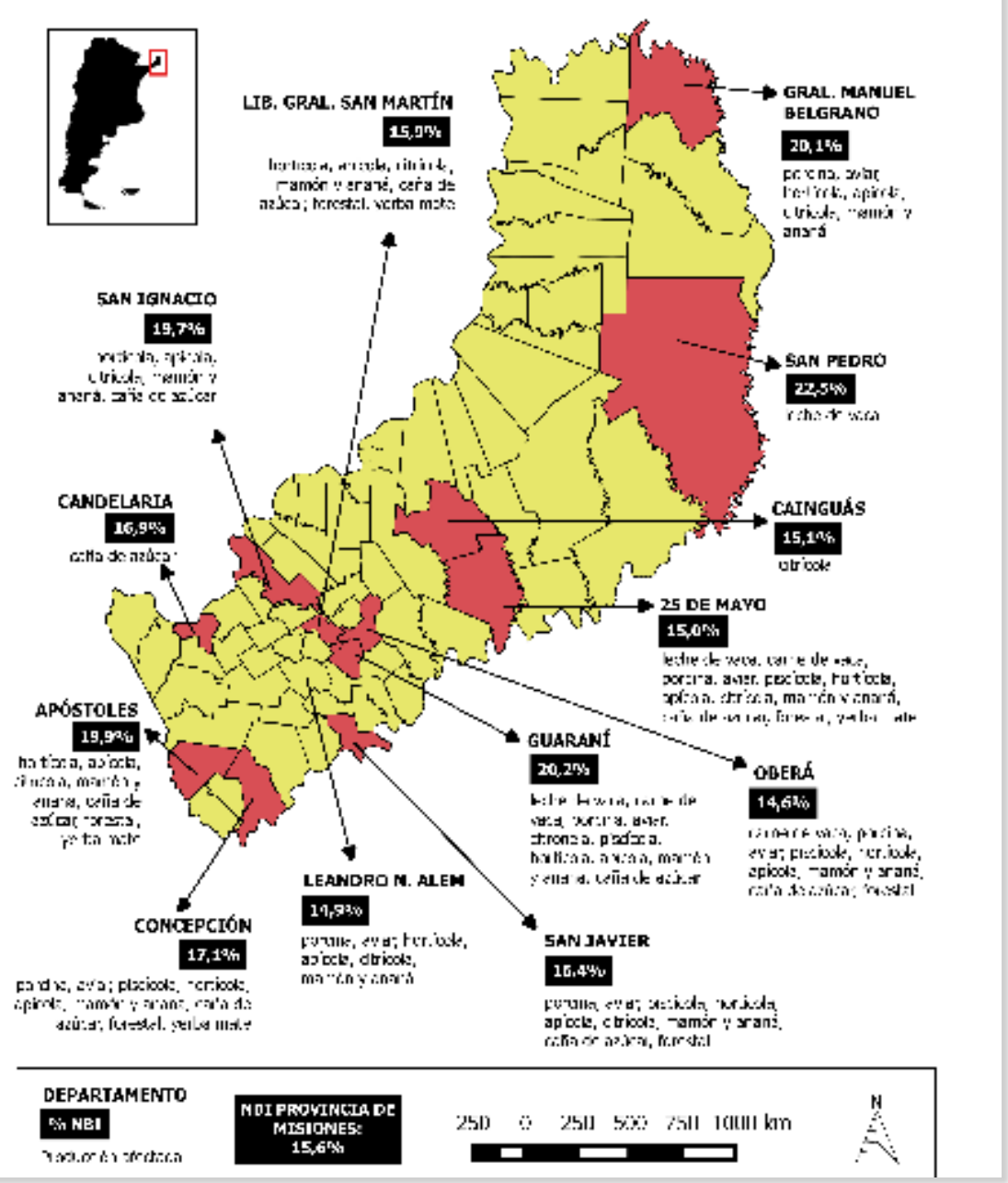

Fuente: Resolución 804/2014: https://www.boletinoficial.gob.ar/detalleAviso/primera/115300/20141114?busqueda=1 Ministerio de Economía (2014): http://www2.mecon.gov.ar/hacienda/dinrep/Informes/archivos/NBIAmpliado.pdf

El último registro periodístico de la declaración de Emergencia y/o Desastre Agropecuario por el Ministerio del Agro yla Producción-MAyP, fue en octubre del 2016, por las tormentas de granizo y fuertes vientos ocurridos durante el mes de julio. Se declaró el estado de emergencia entre agosto de 2016 hasta diciembre del siguiente año, medida tomada a raíz del trabajo conjunto de la Secretaría de Coordinación y Desarrollo Territorial y la CNEyDA, en el marco del SNPyMED (Misiones Online, 18/11/2016). Sin embargo, recién en julio de 2017 los productores recibieron insumos y herramientas por casi \$ 4 millones, como un adelanto de la atención pública ante esta emergencia (Mapa 3).
En esta oportunidad, el titular de la cartera de agricultura de la provincia, se refirió además a la "imposibilidad de entregar dinero en efectivo", razón por la cual los productores se vieron obligados a elegir qué recursos pedir, optando la mayoría por elementos acorde a la actividad que desarrollan. Además, se obsequió "una motosierra, una motoguadaña, ocho bolsas de fertilizantes, ocho de urea, ocho de fosfato y un bidón de glifosato" (Infocampo 12/07/2017).

En los últimos días del año 2018 la Subsecretaría de Desarrollo y Producción Vegetal, dependiente del MAyP de la provincia, respondió ante la emergencia, en diferentes zonas por la ocurrencia de fuertes tormentas con ráfagas de vien- 
to que superaron los $100 \mathrm{~km} / \mathrm{h}$ y caída de granizo (El Territorio, 27/12/2018). En esta ocasión, una vez ocurrido el evento en Aristóbulo del Valle, las autoridades de esta Subsecretaría distribuyeron plásticos, mientras que el gobierno municipal entregó postes para los invernaderos afectados, en los que se producían pimientos y tomates, y donde las pérdidas llegaban a unos \$200 mil. Además, unas cien chacras más fueron afectadas por una tormenta de granizo que provocó pérdidas totales en la producción de tabaco, entre el 40 y $50 \%$ de pérdida en yerba mate y cultivos de sandía y mandioca ${ }^{11}$. Hasta junio de 2019 no fueron registradas en las publicaciones periodísticas con acciones provenientes del ámbito nacional.

11 Hasta el momento de la escritura de este artículo no se encontraron instrumentos legales (boletines oficiales de la $\mathrm{Na}$ ción o de Misiones) que pudieran confirmar esta declaración.

Mapa 3. Declaración de Emergencia Agropecuaria en Misiones en 2016

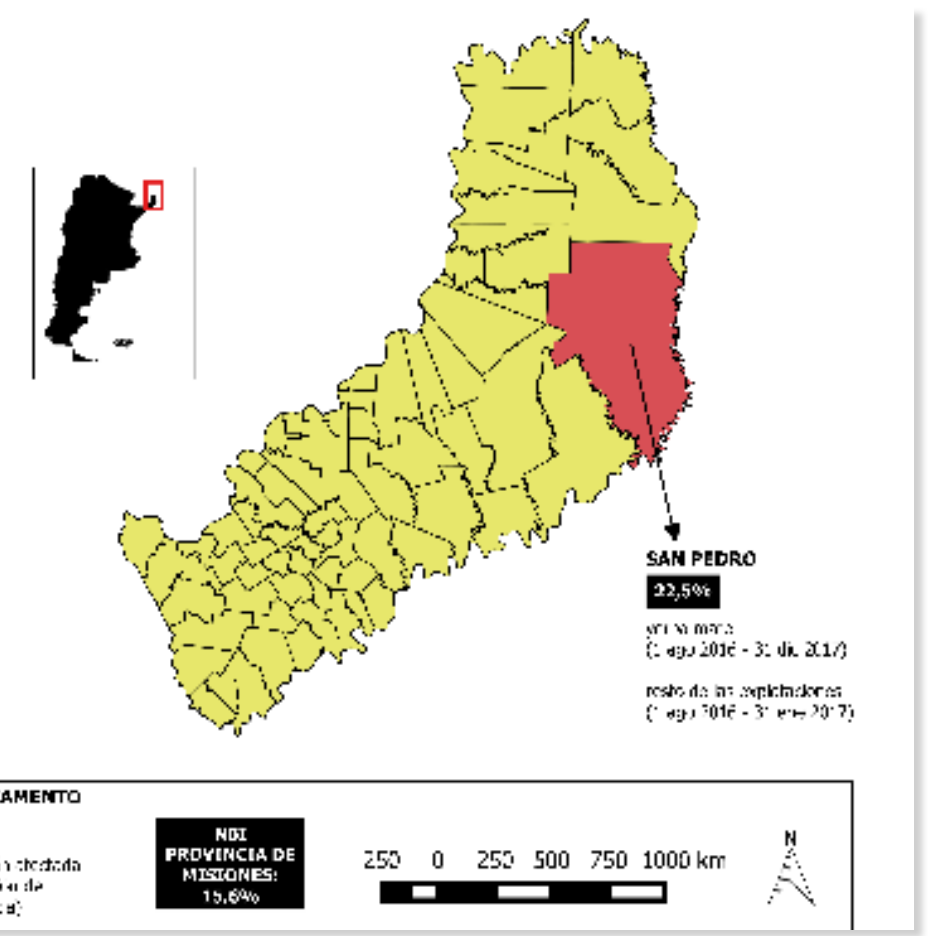

Fuente: Resolución 376 - E/2016: https://www.boletinoficial.gob.ar/detalleAviso/primera/154224/2016118?busqueda=1 Ministerio de Economía (2014): http://www2.mecon.gov.ar/hacienda/dinrep/Informes/archivos/NBIAmpliado.pdf

\section{GR EN LAS ZONAS URBANAS DE MISIONES}

El 20 de diciembre de 2012 fueron afectados más de 20 barrios de Posadas por un temporal de lluvia que marcó un récord de pp, superando el promedio histórico del mes $(206 \mathrm{~mm}$ que representan el $20 \%$ de lo que llueve en todo el año en la provincia). El temporal causó graves problemas, reportándose daños en viviendas y cortes en la provisión de energía eléctrica. La OPAD había anticipado un pronóstico con intensas lluvias, granizo, tormentas eléctricas y fuertes vientos; y recomendó ordenar las prioridades en los pedidos de ayuda según la gravedad de cada situación (riesgo de vida, riesgo de derrumbe, falta de agua y comida y falta de ropas). Se realizaron algunas "tareas momentáneas para limpiar algunos desagües", y se atendió más de "70 familias que perdieron prácticamente todo" (El Territorio, 20/12/2012).

En el 2013 se anunciaron posibles evacuaciones en las provincias de Misiones, Corrientes, Chaco y Santa Fe por la crecida de los ríos Paraná e Iguazú. Ya estaba anegada la Ruta Nacional $N^{\circ} 12$ y se interrumpió el tránsito hacia Puerto Iguazú. “...el incremento verti- 
ginoso del nivel del río es el más importante de los últimos 16 años, con el agravante de la gran cantidad de troncos y basura que arrastran las aguas, lo que obligó a efectuar evacuaciones en las localidades de Iguazú, Puerto Rico y Montecarlo" (La Nación, 26/6/2013).

El 11 de junio de 2014, se publicó que "más de 50.000 personas permanecen aisladas en $\mathrm{Mi}$ siones debido al corte de varios puentes sobre la ruta nacional 12 por la impresionante creciente del río Iguazú, que desde el lunes afecta a toda la cuenca del Alto Paraná". La Policía y Gendarmería cortaron el tránsito en los puentes sobre los arroyos Parana-í, Piray Guazú y el viaducto sobre el Cuñá Pirú (100 km al norte de Posadas). Se anunció también que se esperaban más lluvias para los próximos días por lo cual la situación podía empeorar (Clarín, 11/06/2014).

En los últimos días del mismo mes volvieron a ocurrir inundaciones en el noreste y en Misiones fueron evacuadas más de 7 mil personas en 10 localidades, luego del desborde del Uruguay e Iguazú; se cortaron varios puentes en la ruta 2 y 103, desde San Javier hasta Mosconi. El gobernador anunció la firma del decreto de emergencia y estimó las pérdidas en $\$ 500$ millones (Infobae, 30/6/2014). También se reunió el Comité de Crisis provincial (titulares de las carteras de Salud, Gobierno y Desarrollo Social) y los intendentes de las localidades más afectadas de El Soberbio, Alba Posse, San Javier y Colonia Aurora, para asistir a las familias afectadas y redactar un informe sobre la situación (Visitemos Misiones, 29/06/2014).

Días después nuevamente se produjeron inundaciones por intensas lluvias en Corrientes, Misiones, Chaco y Formosa. Nación anunció el aumento de la asignación universal por hijo, por embarazo, por desempleo, las asignaciones familiares, y del PROGRESAR ${ }^{12}$, por tres meses; lo que benefició a más de 14.000 afectados en

12 Programa de Respaldo a Estudiantes de Argentina (PROG.R.ES.AR) puesto en marcha en el 2014 y todavía continúa, implementado por la Ansés (Administración Nacional de la Seguridad Social), para generar oportunidades de inclusión social y laboral para jóvenes por medio de la promoción de la educación superior y/o formación y capacitación laboral. las cuatro provincias. Se plantea el trabajo articulado con las Fuerzas de Seguridad, Fuerzas Armadas, gobiernos provinciales, municipalidades, Ministerio de Desarrollo Social, la Jefatura de Gabinete y con todas las áreas del gobierno relacionadas con la emergencia y aclaran "que no va a ser solamente esta ayuda ahora en la emergencia, sino también posteriormente en todo lo que sea infraestructura, fundamentalmente en viviendas que hayan sido dañadas". Los departamentos que reciben esta asistencia nacional en Misiones son: San Javier, El Soberbio, Alba Posse, Colonia Aurora, paraje El Saltito y Panambí (CFKArgentina, 02/7/2014).

El 2 de septiembre de 2014 el diputado por Misiones solicitó al gobierno nacional responder a la necesidad de obras sobre todo en la Ruta Nacional No 12 en el tramo Posadas-Iguazú, con la reparación y/o reconstrucción de cinco puentes sobre los arroyos Cuña Pirú, Itacuruzú, Caraguatay y Piray Guazú; y el Río Paranay Guazú (Misiones Online, 02/9/2014).

En enero de 2015 hubo inundaciones que causaron la crecida de arroyos, cortes de energía eléctrica y evacuación de 200 pobladores, con 4 víctimas fatales. La zona más afectada fue la zona sur, la ciudad de Oberá, Posadas, Eldorado, Piray, Montecarlo y Jardín América. El gobernador también organizó una reunión del Comité de Crisis, para atender la situación de los 30 municipios afectados (Minuto Uno (03/01/2015).

Un dato de suma importancia ante la situación de inundación es el riesgo sanitario y desde Salud Pública, en este caso ante la muerte de seis personas, se dio el alerta sobre control de la calidad del agua en las zonas sin acceso a la red de agua potable y se “...recomienda hervir el agua porque con las inundaciones suben las napas y se potencia la posibilidad de cuadros de gastroenteritis" (El Territorio, 05/01/2015).

En noviembre de 2015 se registraron nuevamente fuertes e incesantes lluvias que afectaron a las localidades de San José, Leandro N. Alem y Oberá. Cientos de personas fueron evacuadas, y se produjeron daños materiales y edilicios. La municipalidad de Oberá recibió ropas, calza- 
dos, alimentos y otros elementos para ser distribuidos entre las familias que sufrieron pérdidas materiales (El Tribuno, 11/11/2015).

El 26 de diciembre de 2015 se registraron nuevas inundaciones en el NEA y se anunció que el efecto de la corriente del Niño va a generar lluvias importantes en enero, febrero y marzo, lo que causaría la crecida de ríos y arroyos. Desde el Gobierno Nacional se destacó que las obras de infraestructura es una "asignatura pendiente" en la zona, pero que se están “...enviando los elementos que necesitan los evacuados; Gendarmería y Prefectura también están ayudando en la evacuación (...) los recursos importantes en términos económicos van a ser para la recuperación de lo que deja la creciente". También se organizó un Comité de Crisis con representantes del gobierno nacional y provincial (Casa Rosada Presidencia 26/12/2015).

El 13 de noviembre del mismo año se registró una inundación por el desborde de los arroyos de Alem, que afectó a doscientas familias, que fueron asistidas con agua potable, ropas, alimentos y alojamiento de los evacuados. "La situación en el Litoral sigue siendo alarmante" y se registran "pérdidas irrecuperables" (Misiones Cuatro, 13/11/2015).

En febrero de 2017 se produjeron intensas pp, con granizo y fuertes vientos que afectaron principalmente a Posadas en donde 40 familias de la chacra 181 perdieron sus casas y a las que Desarrollo Social del municipio, les iba a entregar chapas, colchones y frazadas, al igual que a los afectados de San Vicente, Tres Capones, Apóstoles, Gobernador Roca, Azara, San Javier, Santa María y Concepción y Campo Ramón, según información suministrada por la Dirección de Defensa Civil (El Territorio, 22/2/2017).

El 10 de abril del mismo año, el intendente de Capioví y el Director de Defensa Civil confirmaron que tuvieron que mudar la municipalidad por el anegamiento del inmueble y que también hay 25 familias evacuadas en Campo Ramón (Misiones Cuatro, 12/6/2017).

Tras las inundaciones de mayo de 2017, los vecinos del barrio M. Lanús de Posadas, ma- nifestaron su descontento por los problemas ocasionados por la inundación y el lodazal que se formó a raíz de las intensas lluvias de las últimas horas y por falta de mantenimiento y limpieza de los canales de desagüe por parte de la municipalidad. En ese mismo mes también se registraron reclamos de la misma índole en varios barrios de la Capital misionera como en El Porvenir (por desborde de arroyos que afectaron a más de 200 familias); San Lucas (por falta de un sistema de drenaje pluvial, con las tubería, alcantarillas e instalaciones complementarias que permitan el rápido escurrimiento) (Misiones Cuatro 24/5/2017).

El 12 de junio de 2017 en El Soberbio se enunció la creación de una "zona roja para evitar inundaciones", luego de la crecida del Río Uruguay y la necesidad de evacuar a 18 familias. El municipio definió la "zona roja" en la que se "evitará" la construcción de viviendas. El dato fue confirmado por "el Intendente de El Soberbio (...) quien también adelantó que las familias evacuadas volvieron a sus casas (...) y que 18 viviendas serían relocalizadas a una altura de $1.30 \mathrm{~m}$ por encima del nivel del río para evitar futuros problemas con la creciente..." (Misiones Cuatro, 12/6/2017). Sin embargo, no se encontraron noticias posteriores que confirmaran la existencia actual de dicha zona.

También se registraron las inclemencias del tiempo en Posadas, Oberá y Alem, el 13 de noviembre de 2018, lo cual llevó a la Policía de Misiones y la Subsecretaría de Protección Civil, a realizar tareas de relevamiento en toda la provincia, confirmándose la crecida de arroyos, daños en varias casas, caídas de árboles, postes con cables de luz y telefonía, siendo el último de estos departamentos el más afectado, pidiéndose el alerta para Misiones en los organismos provinciales y nacionales (El Territorio, 13/11/2018).

En octubre se produjo una fuerte tormenta eléctrica con intensas lluvias y granizo, que afectó a casi 300 familias que debieron ser asistidas en Posadas y Garupá. Registrándose el desborde de los arroyos La Chancha, Itá, Vicario y Antonica, entre otros. En este contexto se inunda- 
ron varios establecimientos educativos, edificios y viviendas. En noviembre la situación volvió a ser complicada en la provincia. “...desde el Plan Nacional para la Reducción de Desastres Naturales remarcaron que se manejan dos factores, por un lado, el aumento de las amenazas de origen climático y (...) un incremento de la vulnerabilidad de las comunidades frente a las amenazas naturales..." (El Territorio, 13/11/2018).

Para finalizar con esta revisión llegamos al 2018 y principios del año 2019, y según información periodística el 2 de enero 2019 desde la OPAD se anunció la probabilidad de ocurrencia de tormentas periódicas y precipitaciones intensas en los meses de verano (Primera Edición, 31/12/2018, 10/01/2019). Efectivamente, el lunes 7 de enero se publicó la ocurrencia de estos eventos que causaron daños en el centro y sur de la provincia. Dos días de intensas lluvias en el departamento de San Pedro, causaron desbordes de arroyos y zonas inundadas. La Cámara Regional de Comercio, Industria, Producción y Servicio, solicitó "la gestión e inversión para la construcción y reparación de varias obras públicas sobre las principales avenidas de la capital...". (El Territorio, 09/01/2019).

\section{CONCLUSIONES FINALES}

Como se expuso hasta aquí, en lo que respecta a la GR, Misiones posee un plan de emergencia provincial, adherido al SINAGIR, con la finalidad de prevenir desastres, basado en la alerta temprana y la protección civil. Esto último, en el marco de la Subsecretaría de Protección Civil que se encarga de resguardar a la población ante la posibilidad de ocurrencia de un desastre, por medio de la Gestión Integral de Riesgos. Esta Subsecretaría junto al Instituto Provincial de Loterías y Casinos Sociedad del Estado, dispone del PAC dirigido a las familias afectadas por eventos climáticos extremos, que consiste en el otorgamiento de créditos para la compra de muebles y electrodomésticos. Lo que puede servir de marco para afirmar que existe la intensión (al menos en la letra) de prevenir y mitigar, principalmente desde la Subse- cretaría. Al mismo tiempo, también se hace evidente la importancia que se le da a la respuesta al poner en marcha el PAC con el otorgamiento de créditos, luego de la comprobación de afectación económica, con obligación de devolución del dinero más intereses.

Es importante recordar también que en la página web de esta Subsecretaría los afectados pueden hacer su denuncia, para poder recibir diferentes tipos de asistencia. $Y$ allí también pueden buscar asesoramiento, acerca de lo que deben hacer ante la ocurrencia de inundaciones, tormentas, incendios, entre otros, consultando los protocolos disponibles.

Existe el Plan Provincial de Emergencias y Desastres Climáticos para definir, planificar y articular las acciones de prevención, mitigación, respuesta, rehabilitación y reconstrucción. Cabe también mencionar que en la ciudad de Posadas existe la OPAD junto con el SAT. La OPAD centraliza y coordina los informes provenientes de instituciones públicas y privadas, nacionales y extranjeras, y es la única dependencia pública con SAT, lo que la hace responsable de la respuesta en el momento en que ocurre un evento, así como también la que debe promover, planificary mantener la coordinación y el trabajo conjunto.

En el nivel sectorial el SNPyMED se constituye en la principal herramienta de GR para el agro con cobertura en todo el país que incorpora la prevención y la mitigación de los desastres, integrando medidas correctivas y prospectivas de GR; así como también planteando la asistencia a explotaciones familiares o empresariales. La existencia de este Sistema Nacional es sumamente importante, sobre todo para un país con un sector agropecuario tan desarrollado, aunque todavía no ha superado la situación de respuesta, luego de la ocurrencia de eventos extremos. En el período analizado en Misiones, observamos que en lo referente a la declaración de emergencia agropecuaria, su actuación es expos, una vez presentadas las declaraciones juradas (de los damnificados y autoridades locales), que justifican el pedido de emergencia y/o desastre por parte de los diferentes niveles de la administra- 
ción pública (municipio, provincia y nación). En este caso se recurre a la asistencia con la distribución en general de víveres, agua potable, ropa y colchones, así como también a la evacuación de los afectados en caso de ser necesario.

En estas normativas también se formula la atención de la prevención y se establece la constitución de fondos especiales o la designación de los organismos públicos que se deben hacer cargo del costo de obras estructurales, fundamentalmente.

En referencia a la información periodística llaman la atención las noticias en las que las autoridades provinciales, manifiestan la falta de recursos económicos y la contribución pública con el 'regalo' de algunas herramientas, algunos plásticos y postes para reparar unos pocos invernaderos. Así como también, algunas medidas tomadas por las autoridades locales, que se podría decir que plantean, al menos la intención de actuar sobre la vulnerabilidad social. Por ejemplo, con el aumento de la asignación universal por hijo, por embarazo, por desempleo, las asignaciones familiares, y del PROGRESAR; y con la delimitación de la zona roja en El Soberbio, que impediría el asentamiento de población en terrenos bajos ${ }^{13}$.

También resultan de interés los reclamos efectuados por la Cámara de Comercio y la población de diferentes zonas afectadas, por la concreción de las obras de reconstrucción propuestas, evidenciando la falta de cumplimiento de las mismas, por parte de las autoridades competentes.

En este contexto provincial y desde la perspectiva social del riesgo, se puede afirmar que las inundaciones en Misiones se producen por eventos registrados periódicamente (fuertes lluvias y crecidas de ríos), constituyéndose en catástrofes o desastres también habituales, definidos por la vulnerabilidad social, que amplía cada vez más el

13 Este tipo de medida es un solo ejemplo, sobre el cual no se han publicado novedades en ningún periódico hasta la actualidad. riesgo. En este caso por las características de las normativas existentes y su forma de aplicación, así como también por la falta de prevención (al no mejorar la situación económica y social general de la población, al no planificar el desarrollo territorial en función de la vulnerabilidad y estos eventos), las inundaciones se registran y causan cortes de rutas, que dejan incomunicada a la población, producen el anegamiento de viviendas generando una numerosa cantidad de evacuados, la pérdida de producciones familiares, todo lo cual afecta en forma negativa la situación de vulnerabilidad ya existente. En esta realidad también queda manifiesta la distancia que existe entre lo que se enuncia en la letra de las leyes y la aplicación de las mismas ante la ocurrencia de las inundaciones. Se habla de la vulnerabilidad, se la incluye en los cuerpos normativos, pero citando nuevamente a Beck (2008), la suma de las inacciones que ocurren cotidianamente en el territorio (en este caso Misiones), constituye un proceso que conduce a la generación del riesgo de inundaciones que no se anticipa al desastre que las mismas provocan. En nuestra sociedad los procesos socio-territoriales diferencian los riesgos sobre todo entre los diferentes agentes, aún entre aquellos que se ven afectados por estos eventos extremos, que no son incluidos en la normalidad, sino que son siempre interpretados como extremos inusuales, aunque si se recurre a las estadísticas meteorológicas se comprobará lo contrario.

Finalmente, se reitera que es fundamental incorporar los aspectos o causas de fondo de la construcción de las situaciones de vulnerabilidad que definen el desastre, la inundación, ya que en la actualidad, se reconoce el incremento del grado de exposición de las personas y los bienes, y muy poco avance sobre lo que es y cómo se podría reducir la vulnerabilidad, lo cual genera cada vez nuevos, más y mayores riesgos. 


\section{REFERENCIAS}

Banco Mundial, B. (2016). Análisis Ambiental del País. Buenos Aires: Argentina.

Barrenechea, J.; Gentile, E.; González, S. y Natenzon, C. (2000). Una propuesta metodológica para el estudio de la vulnerabilidad social en el marco de la teoría social del riesgo. IV Jornadas de Sociología. FCS, UBA, 6 al 10 de noviembre.

Beck, U. (2008). La sociedad del riesgo mundial. En busca de la seguridad perdida. Barcelona: Paidós.

Blaikie P.; Cannon T.; David I. y Wisner, B. (1996). Vulnerabilidad. El entorno social, político y económico de los desastres. Red de Estudios Sociales en Prevención de Desastres en América Latina, Primera Edición: Julio de 1996

Craviotti, C. (2014). Agricultura familiar en Latinoamérica: continuidades, transformaciones y controversias. Buenos Aires: CICCUS.

Natenzon, C.E. (2005). Vulnerabilidad social, catástrofes y cambio climático. Comentarios temáticos, teóricos y metodológicos para América Latina. II Regional Conference on Global Change: South America. Universidad de San Pablo, Brasil, 7-10 de noviembre.

Obschatko, E. (2009). Las explotaciones agropecuarias familiares en la República Argentina. Un análisis de los datos del Censo Nacional Agropecuario 2002. Buenos Aires: PROINDER.

Presidencia de la Nación (02 de junio de 2014). Asistencia del Estado a damnificados por las inundaciones en Misiones, Formosa, Chaco y Corrientes. Recuperado de https://www.cfkargentina.com/ medidas-para-los-damnificados-por-las-inundaciones-en-misiones-formosa-chaco-y-corrientes/

UNISDR (2015). Marco de Sendai para la reducción del riesgo de desastres 2015-2030. Ginebra.

\section{OtRAs Fuentes}

Casa Rosada Presidencia (2015). Recuperado de https://www.casarosada.gob.ar/informacion/que-estamos-diciendo/35737-inundaciones-en-el-nea

Casa Rosada Presidencia (26/12/2015). Inundaciones en el NEA. Recuperado de https://www.casarosada.gob.ar/informacion/ que-estamos-diciendo/35737-inundaciones-en-el-nea

Dirección Nacional de Relaciones Económicas con las Provincias (DINREP) (2014). Necesidades Básicas Insatisfechas (NBI), Versión ampliada con datos departamentales, Subsecretaría de Relaciones con Provincias, Ministerio de Economía y Finanzas Públicas de la Nación. Recuperado de http://www2.mecon. gov.ar/hacienda/dinrep/Informes/archivos/ NBIAmpliado.pdf

Gobierno de Misiones (03/10/2018). Recuperado de www.misiones.gov.ar

InfoLEG -Información Legislativa Ley 26509, Honorable Congreso de la Nación Argentina, 20 de agosto de 2009 http://servicios. infoleg.gob.ar/infolegInternet/verNorma. do?id=157271

InfoLEG -Sistema Nacional para la Gestión Integral del riesgo y la Protección Civil. Ley 27287. Creación http://servicios.infoleg.gob.ar/infolegInternet/anexos/265000-269999/266631/ norma.htm

Mendoza Gobierno, Defensa Civil (2018). Recuperado de http://www.defensacivil.mendoza. gov.ar/noticias/el-sinagir-presento-los-escenarios-de-riesgo-de-desastre-para-los-proximos-meses/

Ministerio de Seguridad (13/03/2018). Misiones está cerca de adherir al SINAGIR. Recuperado de https://www.argentina.gob.ar/noticias/misiones-esta-cerca-de-adherir-al-sinagir

Oficina de Prevención de Riesgos ante Fenómenos Naturales. http://opad.posadas.gov.ar

Servicio Meteorológico Nacional SMN (s/f). Atlas Climático Argentina. Recuperado de https://www.smn.gob.ar/caracterizacion-estadisticas-de-largo-plazo

Periódicos de Misiones

El Territorio (20/12/2012). Lluvia récord dejó a Posadas bajo agua y en alerta por nuevo diluvio. 
Recuperado de https://www.elterritorio. com.ar/lluvia-record-dejo-a-posadas-bajo-agua-y-en-alerta-por-nuevo-diluvio7346730353751046-et

El Territorio (15/11/2014). Nación declaró la emergencia agropecuaria en la provincia. Recuperado de https://www.elterritorio.com.ar/nacion-declaro-la-emergencia-agropecuaria-en-la-provincia-4657056164312319-et

El Territorio (05/01/2015). Advirtieron sobre riesgo sanitario post inundaciones de los arroyos. Recuperado de https://www.elterritorio.com.ar/ nota4.aspx?c $=3517058195111213$

El Territorio (22/2/2017). Granizada, Iluvia y vientos dejaron sin luz a Itacaruaré. Recuperado de https://www.elterritorio.com.ar/granizada-Iluvia-y-vientos-dejaron-sin-luz-a-itacaruare-6824103581665890-et

El Territorio (26/6/2017). Barrios de Eldorado inundados con torrencial lluvia. Recuperado de https://www.elterritorio.com.ar/ barrios-de-eldorado-inundados-con-torrencial-Iluvia-2579164993141289-et

El Territorio (14/10/2018). En Misiones las tormentas están superando todas las previsiones. Recuperado de https://www.elterritorio.com. ar/en-misiones-las-tormentas-estan-superando-todas-las-previsiones-5223-et

El Territorio (13/11/2018). Renovaron la alerta meteorológica por tormentas intensas para Misiones. Recuperado de https://www.elterritorio.com.ar/ renovaron-la-alerta-meteorologica-por-tormentas-intensas-para-misiones-9133-et

El Territorio (27/12/2018). Productor de tomates y morrones perdió 200 mil pesos por las tormentas. Recuperado de https://www.elterritorio.com. ar/productor-de-tomates-y-morrones-perdio200-mil-pesos-por-las-tormentas-14345-et

El Territorio (09/01/2019). Solicitan reparación de obras públicas en pleno centro de San Pedro. Recuperado de https://www.elterritorio.com.ar/ solicitan-reparacion-de-obras-publicas-enpleno-centro-de-san-pedro-15709-et

El Tribuno (11/11/2015). Varias localidades de Misionesinundadas. Recuperado de https://www.eltri- buno.com/jujuy/nota/2015-11-11-1-30-0-varias-localidades-de-misiones-inundadas

Minuto Uno (03/01/2015). Cuatro muertos y una niña desaparecida por la inundación en Misiones. Recuperdo de https://www.minutouno.com/notas/349252-cuatro-muertos-y-una-nina-desaparecida-la-inundacion-misiones

Misiones Cuatro (13/11/2015). Inundaciones en Alem. Recuperado de https://www.youtube. com/watch?v=uYAB5X9BYBE

Misiones Cuatro (24/5/2017). Inundaciones y calles intransitables en Miguel Lanús, tras el temporal. Recuperado de https://misionescuatro.com/posadas/inundaciones-calles-intransitables-miguel-lanus-tras-temporal/

Misiones Cuatro (12/06/2017). El Soberbio: crean zona roja para evitar inundaciones. Recuperado de https://misionescuatro.com/provinciales/soberbio-crean-zona-roja-evitar-inundaciones/

Misiones Online (18/11/2016). Nación declaró la emergencia agropecuaria en Misiones. Recuperado de https://misionesonline.net/2016/11/18/ nacion-declaro-la-emergencia-agropecuaria-en-misiones/

Misiones Online (19/06/2018). La adhesión a la ley de Gestión del Riesgoyla Protección civil permitirála asistencia automática de Nación en casos extremos. Recuperado de https://misionesonline.net/2018/06/19/ la-adhesion-la-ley-gestion-del-riesgo-la-proteccion-civil-permitira-la-asistencia-automatica-nacion-casos-extremos/

Misiones Online (02/9/2014). Nación tiene en carpeta la reparación de cinco puentes en Misiones. Recuperado de https://misionesonline. net/2014/09/02/nacion-tiene-en-carpeta-la-reparacion-de-cinco-puentes-en-misiones/

Noticias del 6 (20/01/2012). Se avanza en el proceso de la emergencia agropecuaria. Recuperado de https:// www.noticiasdel6.com/se-avanza-en-el-proceso-de-la-emergencia-agropecuaria/

Primera Edición (31/12/2018). Amenaza de chaparrones y tormentas para la última noche de 2018. Recuperado de https://www.primeraedicion. com.ar/nota/100068007/amenaza-de-chaparrones-y-tormentas-para-la-ultima-no- 
che-de-2018/

Primera Edición (10/01/2019). Las tormentas podrían ser muy fuertes hoy en Misiones. Recuperado de https://www.primeraedicion.com.ar/ nota/100071002/las-tormentas-podrian-sermuy-fuertes-hoy-en-misiones/

Visitemos Misiones (29/06/2014). Misiones vive una de las peores inundaciones de su historia. Recuperado de https://www.visitemosmisiones.com/noticias/noticias/misiones-vive-una-de-las-peores-inundaciones-de-su-historia/

\section{Periódicos de CABA}

Clarín (11/06/2014). Crecida record. En Misiones, la inundación ya dejó aisladas a más de 50.000 personas. El río empezó a bajar, pero tres localidades siguen incomunicadas por los cortes en los puentes. Recuperado de https://www.clarin.com/sociedad/ misiones-inundacion-dejo-aisladas-personas_0_B1wpHncPmg.html

Infobae (30/6/2014). Más de 12.000 evacuados por las inundaciones en el noreste del país. Recuperado de https://www.infobae. com/2014/06/30/1577055-mas-12000-evacuados-las-inundaciones-el-noreste-del-pais/

Infocampo (12/07/2017). Productores de Misiones recibieron ayuda por la emergencia agropecuaria de 2016. Recuperado de https://www. infocampo.com.ar/productores-de-misiones-recibieron-asistencia-por-la-emergencia-agropecuaria/

La Nación (26/6/2013). Tras el desborde del río Iguazú, que divide a Argentina de Brasil. Misiones: las inundaciones obligaron a cerrar las cataratas del Iguazú. Recuperado de https://www. lanacion.com.ar/sociedad/se-esperan-inundaciones-en-cuatro-provincias-del-litoral-nid1596167

Sandra Pereira es Magister de la Universidad de Buenos Aires (UBA). Investigadora del Instituto de Geografía "Romualdo Ardissone". Docente del Departamento de Geografía. Facultad de Filosofía y Letras. Universidad de Buenos Aires. Desde 1990 participa en forma continua en proyectos de investigación con sede en el Instituto de Geografía. Las temáticas estudiadas están relacionadas con la geografía rural, la producción agropecuaria pampeana y extrapampeana, las organizaciones sociales, el desarrollo rural y las políticas públicas, los riesgos ambientales, la vulnerabilidad social, la construcción social y gestión de riesgos, entre otros. Desde 1996 es docente del departamento de Geografía, en materias como Geografía Social Argentina, Recursos y Sociedad. Y seminarios sobre la Pequeña producción agraria en la Argentina; Ambiente, cultura y poder: usos y disputas en torno al agua en América Latina; Lecturas Clásicas en Economía; Investigación sobre las prácticas docentes en geografía, entre otros. También se desempeñó como profesora en FLACSO Argentina (2007-2018). Tiene publicaciones en revistas especializadas, capítulos de libros y participaciones en numerosos congresos y encuentros sobre dichas temáticas. Departamento de Geografía. Facultad de Filosofía y Letras. Universidad de Buenos Aires. Puán 480 (1406) Ciudad Autónoma de Buenos Aires, Buenos Aires, Argentina, sanper@filo.uba.ar 